\title{
Deutscher Schmerzpreis
}

\section{Antibiotika gegen Schmerzen}

\author{
Die dänische Wissenschaftlerin Dr. Hanne Albert erhält für ihre \\ Forschung im Bereich der Behandlung von Rückenschmerzen mit \\ Antibiotika in diesem Jahr den Deutschen Schmerzpreis.
}

$\mathrm{D}$ er mit 10.000 Euro dotierte Deutsche Schmerzpreis geht in diesem Jahr in unser nördliches Nachbarland Dänemark. Die Forscherin Dr. Hanne Albert von der University of Southern Denmark wurde für ihre Studien zur Behandlung von Rückenschmerzen mit Antibiotika ausgezeichnet. „Dr. Hanne Albert hat mit ihrer bahnbrechenden Arbeit schmerzmedizinische Denkweisen nachhaltig verändert und eine neue Diskussionsbasis zum Verständnis von chronischen Rückenschmerzen geschaffen“, betonte Dr. Gerhard Müller-Schwefe, Präsident der Deutschen Gesellschaft für Schmerzmedizin (DGS), anlässlich der Verleihung des Deutschen Schmerzpreises im Rahmen des Deutschen Schmerz- und Palliativtages am 24. März 2017 in Frankfurt am Main.

\section{Bakterien in der Bandscheibe}

Etwa die Hälfte der Patienten mit chronischen Schmerzen im unteren Rücken weisen im MRT Ödeme im Knochenmark, sogenannte modic changes, auf. Bei ersten Untersuchungen konnte Albert in mehr als $50 \%$ der nach einem Bandscheibenvorfall entnommenen Gewebeproben Bakterien nachweisen. Zum Großteil war das Gewebe mit P. acnes infiziert. Dieses Bakterium, das zur natürlichen Mundflora gehört, gelangt über kleine Verletzungen, die beim Zähneputzen entstehen, ins Blut. Über neu gebildete Kapillaren an dem ausgetretenen Gewebe der Bandscheibe gelangen die Bakterien schließlich ins Innere der Bandscheibe und verbleiben dort auch nach einer Ausheilung des Bandscheibenvorfalls und verursachen Entzündung, Knochenödem und Schmerzen.

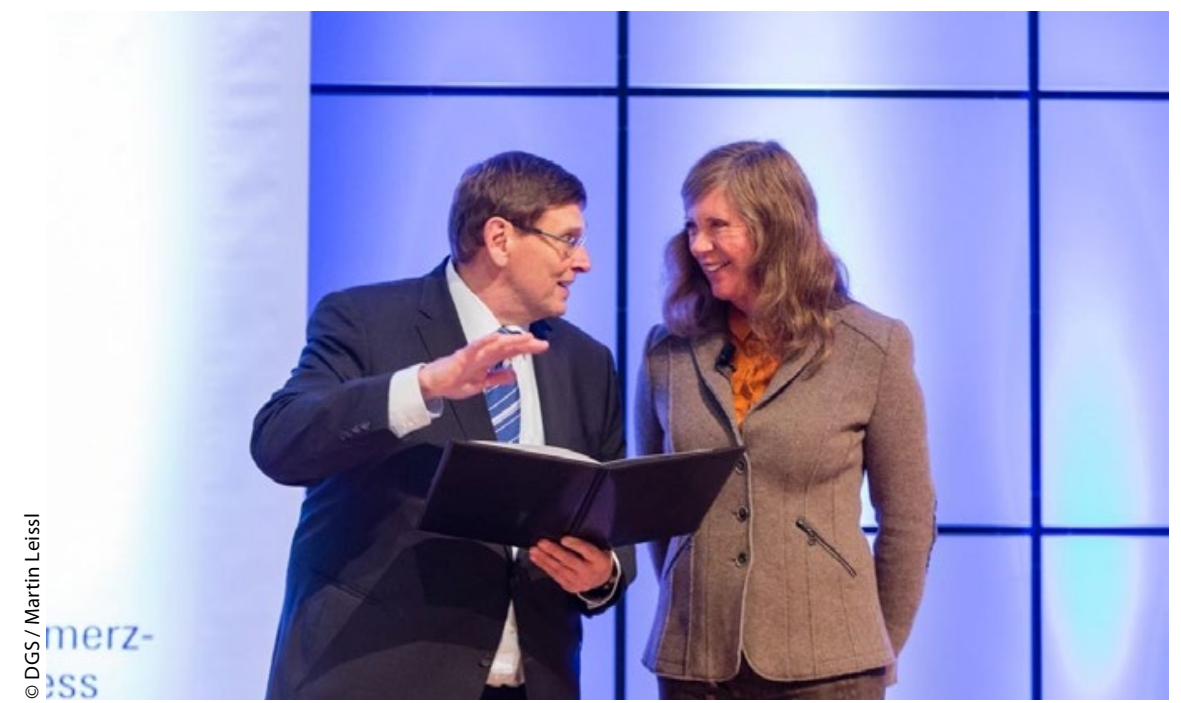

Dr. Gerhard Müller-Schwefe und die Preisträgerin Dr. Hanne Albert bei der Verleihung des Deutschen Schmerzpreises.
So entstand die Idee, in einer Pilotstudie zu testen, ob Antibiotika gegen den Bakterienbefall und damit auch gegen die Rückenschmerzen der Patienten wirken können. Bereits diese erste Studie zeigte signifikante Ergebnisse in der Verbesserung sowohl der Schmerzsymptome als auch der funktionellen Beschwerden der Patienten [Albert HB et al. Br J Sports Med. 2008:42:969-73]. Weitere randomisierte, placebokontrollierte Studien haben das Ergebnis bestätigt [Albert $\mathrm{HB}$ et al. Eur Spine J. 2013;22:697-707; Mohanned A. Al-Falahi et al. The Iraqi postgraduate Medical Journal 2014;13:390-398]. Die Patienten erhielten über einen Zeitraum von 100 Tagen dreimal täglich 1.000 mg Amoxicillin. Erste Effekte zeigten sich nach sechs bis acht Wochen und setzten sich über eine Follow-up-Zeit von einem Jahr, in einer weiteren Studie sogar über zwei Jahre, fort.

Auf die Frage, ob nun alle Patienten mit Schmerzen im unteren Rücken mit Antibiotika behandelt werden sollen, sagte Albert: „Nein, aber diejenigen mit ,modic changes', bei denen Bakterien eine Rolle spielen, profitieren enorm.“

\section{Jährliche Verleihung}

Der Deutsche Schmerzpreis - Deutscher Förderpreis für Schmerzforschung und Schmerzmedizin - wird jährlich an Persönlichkeiten verliehen, die sich durch wissenschaftliche Arbeiten über Diagnostik und Therapie akuter und chronischer Schmerzzustände verdient gemacht oder die durch ihre Arbeit oder ihr öffentliches Wirken entscheidend zum Verständnis des Problemkreises Schmerz und der davon betroffenen $\mathrm{Pa}$ tienten beigetragen haben.

Wissenschaftlicher Träger des Deutschen Schmerzpreises ist die Deutsche Gesellschaft für Schmerzmedizin. Der Preis wird gemeinsam mit der Deutschen Schmerzliga verliehen und von Mundipharma gestiftet. 